\title{
SARS-CoV-2 can induce brain and spine demyelinating lesions
}

\section{Luca Zanin ${ }^{1} \cdot$ Giorgio Saraceno ${ }^{1} \cdot$ Pier Paolo Panciani $^{1}$ (I) $\cdot$ Giulia Renisi $^{2} \cdot$ Liana Signorini $^{2} \cdot$ Karol Migliorati $^{1}$. Marco Maria Fontanella ${ }^{1}$}

Received: 17 April 2020 / Accepted: 23 April 2020 / Published online: 4 May 2020

(C) Springer-Verlag GmbH Austria, part of Springer Nature 2020

\begin{abstract}
SARS-CoV-2 can attack the central nervous system in the early stages of infection. Headache, anosmia, and dysgeusia are common symptoms. Disturbance of consciousness and seizures can occur as complications in case of severe COVID-19. We described the case of a COVID-19 patient admitted for interstitial pneumonia and seizures. MRI showed newly diagnosed demyelinating lesions. High-dose steroid treatment allowed neurological and respiratory recovery. We speculated a delayed immune response induced by SARS-CoV-2. The virus may lead to a SIRS-like immune disorder or play a role of infective trigger. Prompt invasive treatment should be adopted to avoid hypoxic neurotoxicity and prevent CNS injuries.
\end{abstract}

Keywords COVID-19 $\cdot$ SARS-CoV-2 $\cdot$ Demyelinating lesions $\cdot$ Seizures $\cdot$ Immune response $\cdot$ Neuro-COVID

\section{Introduction}

On January 24, 2020, a new virus named Severe Acute Respiratory Syndrome Coronavirus 2 (SARS-CoV-2) has been identified, quickly gaining worldwide attention [21]. More than one third of patients with SARS-CoV-2 develop neurological manifestations [10]. Similarly to other Coronavirus, SARS-CoV-2 can attack the olfactory bulb and then affect the central nervous system (CNS) through the olfactory tract in the early stages of infection [5]. Neurological impairment and demyelinating reaction appear as complications in case of severe Coronavirus Disease 2019 (COVID-19) [10]. We described the case of a COVID-19 patient with newly diagnosed demyelinating lesions.

This article is part of the Topical Collection on Infection

Pier Paolo Panciani

pierpaolo.panciani@gmail.com

1 Neurosurgery, Department of Medical and Surgical Specialties, Radiological Sciences and Public Health, University of Brescia, Spedali Civili di Brescia, Piazzale Spedali Civili, 1, 25124 Brescia, Italy

2 Department of Infection and Tropical Disease, University of Brescia, Brescia, Italy

\section{Case report}

A 54 years old women, with a past medical history of anterior communicating artery (AComA) aneurysm treated surgically 20 years before, was found unconscious at home. When the rescue arrived, she regained consciousness and became unrest. At the emergency department, a brief neurological examination revealed a GCS of 12 (E3 M6 V3), without focal sensorimotor deficits. No signs of both tongue biting and incontinence were reported by the familiars. Anosmia and ageusia were referred by several days. Head CT scan was normal

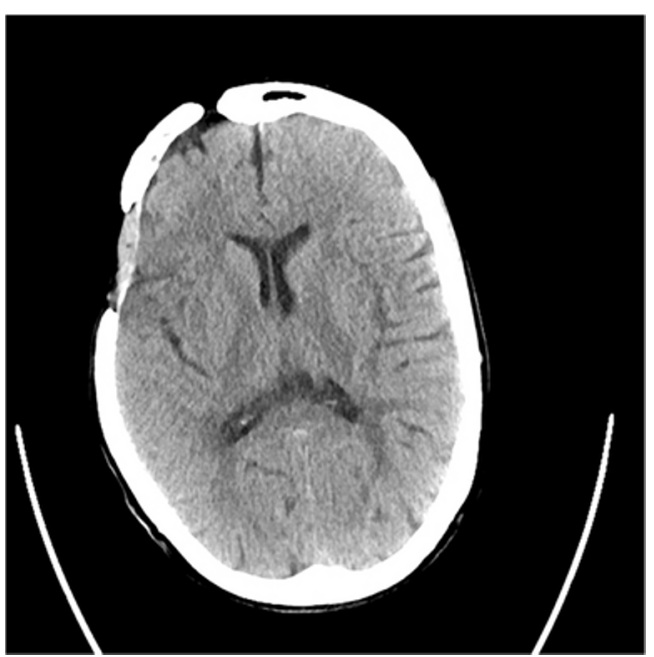

Fig. 1 Head-CT scan: right frontotemporal craniotomy (previous AComA aneurysm surgery). No evidence of acute injuries 
(Fig. 1). Chest X-ray (Fig. 2) revealed an interstitial pneumonia (IP), and real-time polymerase chain reaction (RT-PCR) for SARS-CoV-2 was positive.

The patient was admitted to our Neurosurgical Unit and complete blood tests showed moderate lymphocytopenia with mild elevation of inflammatory indices (WBC $8.81 / \mathrm{mm}^{3}$, Ly $0.3 / \mathrm{mm}^{3}$, CRP $41.3 \mathrm{mg} / \mathrm{L}$, Fibrinogen $520 \mathrm{mg} / \mathrm{dL}$ ). Both blood and urinary cultures were negative. Antiretroviral and hydroxychloroquine were started. No abnormalities at arterial blood gas (ABG) analysis were detected (pO2 89, pCO2 41, $\mathrm{pH}$ 7.43).

After few hours, the patient clinically deteriorated. Body temperature was normal, and no electrolyte disorders were found. ABG revealed a severe normocapnic hypoxia. Therefore, she was intubated. Subsequent head CT scan was unchanged. Electroencephalography showed two seizures starting from right frontotemporal region and diffusing in homologous contralateral hemisphere. Antiepileptic therapy with lacosamide, levetiracetam, and phenytoin was started with seizures control.

Brain MRI revealed alterations of the periventricular white matter, hyperintense in T2WI, without restriction of diffusion nor contrast enhancement (Fig. 3a-f). Similar lesions were found at the bulbo-medullary junction and in both the cervical and dorsal spinal cord (Fig. 3g). Chemical-physical cerebrospinal fluid (CSF) examination was normal, and further analysis ruled out multiple sclerosis. The CSF RT-PCR for neurotropic viruses, including SARS-CoV-2, was negative.

High-dose steroid treatment (dexamethasone $20 \mathrm{mg}$ /die for 10 days and $10 \mathrm{mg} /$ die for 10 days) allowed a progressive recovery of the pulmonary impairment. The patient was tracheostomized on the 7th day. After 15 days, ventilator weaning was performed, and the patient was discharged from the intensive care unit (ICU) and addressed to our Neurosurgical Unit. The patient was transferred to rehabilitation without sensorimotor deficits after 12 days.

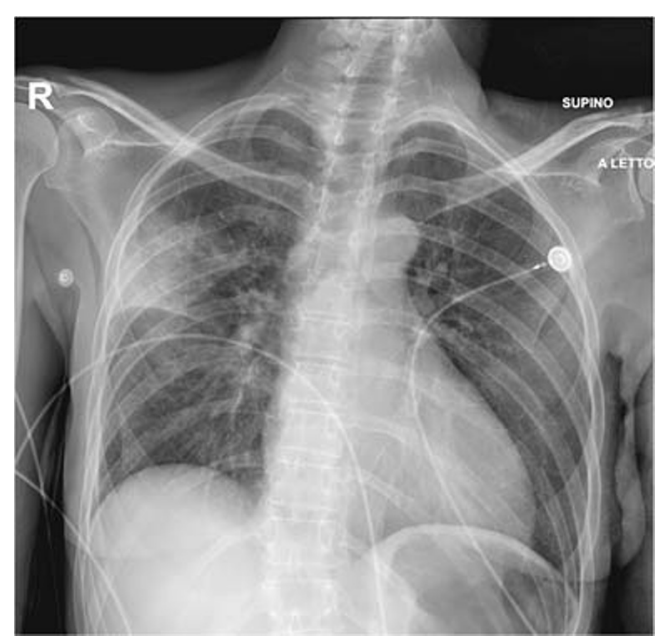

Fig. 2 Chest X-ray: typical COVID-19 interstitial pneumonia

\section{Discussion}

The family of Coronaviruses shows a potential neurotropism that can induce neurological disorders like polyneuropathy, encephalopathy, demyelinating lesions, and ischemic stroke $[8,15]$. The main clinical manifestations are headache, disturbance of consciousness, paralysis, paraesthesia, and seizures [14]. The neurological complications could appear delayed to respiratory symptoms [8].

SARS-CoV-2 shows a genetic similarity to SARS-CoV and MERS-CoV $[4,17]$ and presents an analogous neurotropism. Previous articles showed that a large number of patients report anosmia and dysgeusia. Moreover COVID-19 may lead to symptoms similar to intracranial infections [2].

Our patient showed symptoms consistent with a neurological involvement consequent to SARS-CoV-2 infection. Anosmia and dysgeusia appeared early, while seizures occurred as COVID-19 complication. Moriguchi explains seizures as results of SARS-CoV-2 encephalitis [12]. Otherwise, we observed demyelinating lesions related to the neurological impairment.

The presence of demyelination, as well as SARS-CoV virus particles and genome sequences, in the brain has been detected in autopsy studies [6, 19]. Our patient's brain and spine MRI showed new onset of multiple, non-enhancing demyelinating lesions. Previous cerebral MRI controls performed as follow-up after the AComA aneurysm surgery were normal. Multiple sclerosis (MS), viral encephalitis, and bacterial infections were excluded. Therefore, we speculated a pathogenesis SARS-CoV-2 related.

Neurotropism may occur via trans-lamina cribrosa that enables SARS-CoV-2 to reach the brain through the olfactory tract [1]. The interaction between the spike protein S1 and the host ACE- 2 receptor, expressed in the capillary endothelium, allows the virus to penetrate into the neuronal cells [16]. The viral particles budding lead to the onset of symptoms such as anosmia and dysgeusia in the early phase of infection [10].

The delayed CNS damage appears mediated by the immune system [9]. As previously demonstrated, the pathogenesis of severe viral infections is closely linked to the development of virus induced systemic inflammatory response syndrome (SIRS) or SIRS-like immune disorders [3]. For SARSCoV-2 infection, the pro-inflammatory state induced by the cytokine storm, mainly sustained by IL1, IL-6, and TNF $\alpha$, may be responsible of the activation of glial cells with subsequent demyelination [11]. A possible alternative could be the production of antibodies against glial cells triggered by the virus, as a para-infective or post-infective phenomenon. Zhao described a case report of Guillan-Barrè syndrome during SARS-CoV-2 infection [20]. Moreover, SARS-CoV-2 may play a role of infective trigger, similar to the one of Epstein Barr virus in MS. 


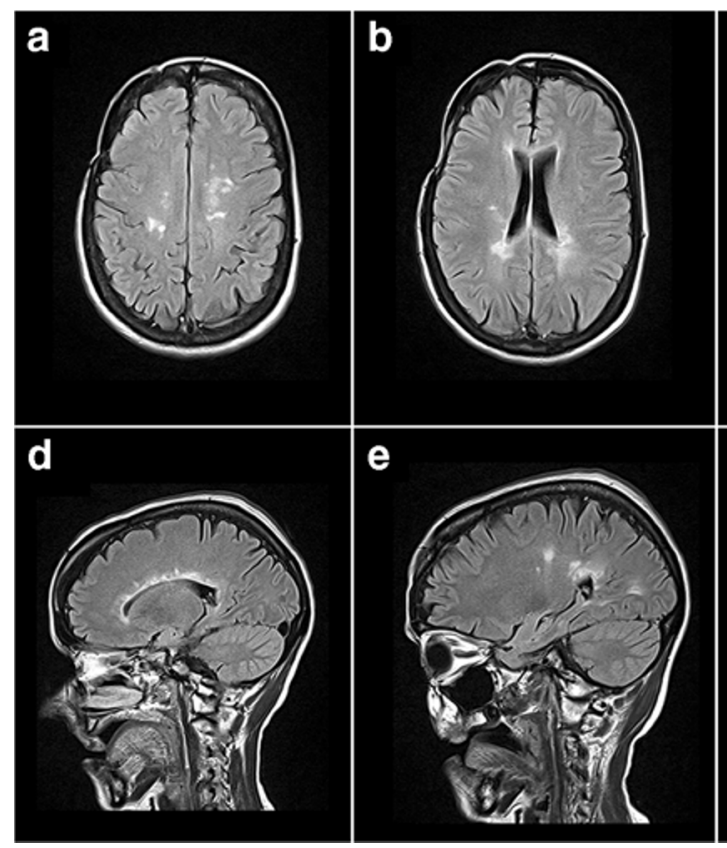

Fig. 3 Head-MRI Flair axial view (a), (b), (c), and sagittal view (d), (e), (f): numerous periventricular white matter alterations, confluent with each other and compatible with demyelinating lesions, adjacent to the temporal, frontal and occipital horns and to the trigones, hyperintense in $\mathrm{T} 2$, without restriction of diffusion and without contrast enhancement;

In SARS-CoV-2 infection, neurological impairment was observed only in case of severe COVID-19 [10]. As a matter of fact, our patient showed an IP that required ICU. Therefore, we supposed that a severe pneumonia with subsequent CNS hypoxia that leads to an increased anaerobic metabolism is required to trigger a neurological damage.

SARS-CoV-2 was not detected in the CSF probably because the neurological damage was sustained by a delayed immune response that occurred after the viremia. Moreover, as reported by different authors, CSF clearance, low sensibility of the method, and delayed sampling could explain this occurrence $[7,13$, $18]$.

\section{Conclusion}

Sudden neurological impairment with seizures in COVID-19 patients may be sustained by CNS involvement and demyelinating lesions. Early CSF collection is suggested. Prompt invasive treatment should be adopted to avoid hypoxic neurotoxicity and prevent CNS injuries.

\section{Compliance with ethical standards}

Conflict of interest The authors declare that they have no conflict of interest.

Informed consent The patient's consent has been obtained for this publication.
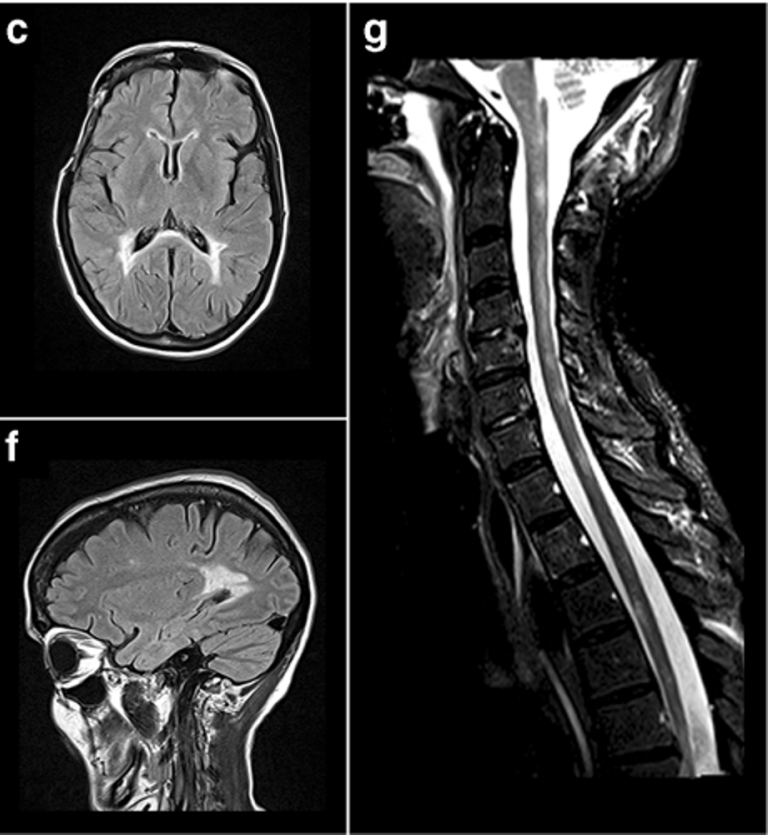

cervical and dorsal MRI T2WI sagittal view (g): numerous focal hyperintense intramedullary signal alterations in $\mathrm{T} 2$ and without contrast enhancement, located at the bulb-medullary junction, at $\mathrm{C} 2$ and from $\mathrm{C} 3$ to Th 6

\section{References}

1. Baig AM, Khaleeq A, Ali U, Syeda H (2020) Evidence of the COVID-19 virus targeting the CNS: tissue distribution, host-virus interaction, and proposed neurotropic mechanisms. ACS Chem Neurosci. https://doi.org/10.1021/acschemneuro.0c00122

2. Caso F, Costa L, Ruscitti P, Navarini L, Del Puente A, Giacomelli R, Scarpa R (2020) Could Sars-coronavirus-2 trigger autoimmune and/or autoinflammatory mechanisms in genetically predisposed subjects? Autoimmun Rev 102524

3. Chen C, Zhang XR, Ju ZY, He WF (2020) Advances in the research of cytokine storm mechanism induced by Corona virus disease 2019 and the corresponding immunotherapies. Zhonghua Shao Shang Za Zhi 36:E005

4. Corman VM, Landt O, Kaiser M et al (2020) Detection of 2019 novel coronavirus $(2019-\mathrm{nCoV})$ by real-time RT-PCR. Eurosurveillance. https://doi.org/10.2807/1560-7917.ES.2020.25. 3.2000045

5. Desforges M, Le Coupanec A, Dubeau P, Bourgouin A, Lajoie L, Dubé M, Talbot PJ (2019) Human coronaviruses and other respiratory viruses: underestimated opportunistic pathogens of the central nervous system? Viruses. https://doi.org/10.3390/v12010014

6. Gu J, Gong E, Zhang B et al (2005) Multiple organ infection and the pathogenesis of SARS. J Exp Med 202(3):415-424

7. Helms J, Kremer S, Merdji H et al (2020) Neurologic features in severe SARS-CoV-2 infection. N Engl J Med. https://doi.org/10. 1056/NEJMc2008597

8. Kim JE, Heo JH, Kim HO, Song SH, Park SS, Park TH, Ahn JY, Kim MK, Choi JP (2017) Neurological complications during treatment of middle east respiratory syndrome. J Clin Neurol 13(3):227233

9. Klein RS, Garber C, Howard N (2017) Infectious immunity in the central nervous system and brain function. Nat Immunol 18(2): 132-141 
10. Mao L, Jin H, Wang $M$ et al (2020) Neurologic manifestations of hospitalized patients with coronavirus disease 2019 in Wuhan, China. JAMA Neurol. https://doi.org/10.1001/jamaneurol.2020. 1127

11. Mehta P, McAuley DF, Brown M, Sanchez E, Tattersall RS, Manson JJ (2020) COVID-19: consider cytokine storm syndromes and immunosuppression [published online ahead of print, 2020 Mar 16]. Lancet S0140-6736(20):30628-0

12. Moriguchi T, Harii N, Goto J et al (2020) A first case of meningitis/ encephalitis associated with SARS-Coronavirus-2. Int J Infect Dis. https://doi.org/10.1016/j.ijid.2020.03.062

13. Panciani PP, Saraceno G, Zanin L, Renisi G, Signorini L, Fontanella MM (2020) SARS-CoV-2: "Three-steps" infection model and CSF diagnostic implication. Brain Behav Immun. [In Press]

14. Saad M, Omrani AS, Baig K et al (2014) Clinical aspects and outcomes of 70 patients with Middle East respiratory syndrome coronavirus infection: a single-center experience in Saudi Arabia. Int J Infect Dis 29:301-306

15. Tsai LK, Hsieh ST, Chang YC (2005) Neurological manifestations in severe acute respiratory syndrome. Acta Neurol Taiwanica 14(3): 113-119

16. Wrapp D, Wang N, Corbett KS, Goldsmith JA, Hsieh CL, Abiona O, Graham BS, McLellan JS (2020) Cryo-EM structure of the
2019-nCoV spike in the prefusion conformation. Science eabb2507. https://doi.org/10.1126/science.abb2507

17. Wu F, Zhao S, Yu B et al (2020) A new coronavirus associated with human respiratory disease in China. Nature 579(7798):265-269

18. Ye M, Ren Y, Lv T (2020) Encephalitis as a clinical manifestation of COVID-19. Brain Behav Immun. https://doi.org/10.1016/j.bbi. 2020.04.017

19. Zhang QL, Ding YQ, Hou JL et al (2003) Detection of severe acute respiratory syndrome (SARS)-associated coronavirus RNA in autopsy tissues with in situ hybridization. Di Yi Jun Yi Da Xue Xue Bao 23(11):1125-1127

20. Zhao H, Shen D, Zhou H, Liu J, Chen S, 2020. Guillain-Barrè syndrome associated with SARS-CoV-2 infecion: causality or coincidence? Lancet Neurol. 2020. https://doi.org/10.1016/S14744422(20)30109-5

21. Zhu N, Zhang D, Wang W et al (2020) A novel coronavirus from patients with pneumonia in China, 2019. N Engl J Med 382(8): $727-733$

Publisher's note Springer Nature remains neutral with regard to jurisdictional claims in published maps and institutional affiliations. 